\title{
About Error Calculation in X-Ray Stress Measurement Balder Ortner
}

\author{
Schillerstrasse 34, Leoben, Austria \\ Balder.Ortner@aon.at
}

Keywords: x-ray stress measurement, error calculation, principal stress, von Mises stress

\begin{abstract}
It is shown that the knowledge of standard deviations $\left(\Delta \sigma_{i j}\right)$ of the components of a stress tensor $\left(\sigma_{\mathrm{ij}}\right)$ is not sufficient to calculate also standard deviations of quantities derived from the stress tensor, as principal stresses $\left(\sigma_{\mathrm{I}}, \sigma_{\mathrm{II}}, \sigma_{\mathrm{III}}\right)$, von Mises stress, Tresca stress, and the components of the deviatoric stress tensor $\sigma_{\mathrm{ij}}^{\prime}$. For such a calculation one needs all information about the measurement and the method for the calculation of $\sigma_{\mathrm{ij}}$. This information is: the accuracy of each measured lattice plane distance and the X-ray elastic factors $\mathrm{F}_{\mathrm{ij}}(\varphi, \psi, \mathrm{hkl})$ of each measured point. Equations are given for the calculation of the standard deviations of all the mentioned quantities. For special cases of measurement strategy the wanted calculations become easier. This is also given.
\end{abstract}

\section{Introduction}

In an X-ray stress measurement, the primary results are components of the stress tensor together with their standard deviations. Further quantities of interest like principal stresses, deviatoric stress, von Mises stress etc. are easily calculated. But it is much more complicated to calculate also the standard deviations for these quantities.

The problem has been tackled in [1], yet not in a general and analytic way but instead by some Monte Carlo simulation. Furthermore, in this paper only the scatter of principal stresses together with the scatter of principal stress axes is investigated. In [2] the error calculation for principal stresses can be found, but in this paper it has been done under the assumption that the traditional $\sin ^{2} \psi$ method is used which, to our opinion, should be regarded as obsolete [6].

All the following considerations and results are done on the basis of the matrix method sometimes we call it also generalized $\sin ^{2} \psi$ method - and are therefore only valid for data obtained by this method. Actually it would be also possible to do the same for virtually all other methods for stress calculation, from $\sin ^{2} \psi$ method to Dölle-Hauk method, to f-method, to $\psi$-integral-method and so on. But it would be much more complicated and we don't think it is necessary.

Our considerations regard only statistical errors, possible errors in the x-ray elastic factors $\mathrm{F}_{\mathrm{ij}}$ are not dealt with since these are systematic errors. If the material to be probed has a sharp texture, these errors can stem from incorrect values of the single crystal elastic parameters $\left(\mathrm{S}_{\mathrm{ij}} / \mathrm{C}_{\mathrm{ij}}\right)$ or inaccurate texture data. In a quasi-isotropic material inaccurate $\mathrm{x}$-ray elastic constants $\left(\mathrm{s}_{1}, \mathrm{~s}_{2}\right)$ would also result in systematic errors of the components of the F-tensor. Inaccurate values of x-ray elastic factors produce inaccurate entries in the Moore-Penrose pseudoinverse and therefore inexact stress values. This is quite similar to errors produces by wrong XEC in the $\sin ^{2} \psi$ method but much more complicated from a mathematical point of view. It is also clear that if one knew the errors in $\mathrm{F}$ and therefore in the pseudoinverse and in $\sigma_{\mathrm{ij}}$, the errors in all the derived stresses can be calculated in the same way as described below.

\section{The Difficulties for "Error Propagation" from $\sigma_{\mathrm{ij}}$ to the Derived Quantities}

There are two obstacles in the error propagation:

a) The quantities $\Delta \sigma_{i j}$ are not independent from each other, because each of them depends in different ways on the standard deviations of the measured values $\Delta \mathrm{d}(\varphi, \psi, \mathrm{hkl})$. Therefore, the simple error propagation by calculation of sums of $\Delta^{2} \sigma_{i j}$ would not be correct. 
b) Neither $\Delta \sigma_{i j}$ nor $\Delta^{2} \sigma_{i j}$ are components of a second rank tensor. Therefore none of these matrices transform like $\sigma_{\mathrm{ij}}$ or $\varepsilon_{\mathrm{ij}}$ does.

\section{The Solution}

Our approach is based on the matrix method together with the concept of the Moore-Penrose pseudoinverse. The matrix method is based on the linear relationship between the measured data $\mathrm{d}(\varphi, \psi, \mathrm{hkl})$ and the wanted components of the stress tensor $\sigma_{\mathrm{ij}}$. This relationship is the well known equation discovered by Dölle and Hauk $[3,4]$.

$$
\varepsilon(\varphi, \psi, \mathrm{hkl})=\mathrm{F}_{\mathrm{ij}}(\varphi, \psi, \mathrm{hkl}) \sigma_{\mathrm{ij}}
$$

Actually it can be regarded as some special form of Hooke's law.

With Dölle-Hauk's equation and knowing the components of the second rank tensor $F_{\mathrm{ij}}(\varphi, \psi, \mathrm{hkl})$ one can establish a system of linear equations, the solution can be written in the form

$$
\sigma_{\mathrm{ij}}=b_{\mathrm{ijn}} \mathrm{y}_{\mathrm{n}} \text {. }
$$

For the moment we use $\mathrm{y}$ as the measured data, y can be either $\varepsilon(\varphi, \psi, \mathrm{hkl})$ or $\mathrm{d}(\varphi, \psi, \mathrm{hkl})$, the measured lattice plane distance or $\mathrm{a}(\varphi, \psi, \mathrm{hkl})$, a fictitious lattice parameter calculated from $\mathrm{d}(\varphi, \psi, \mathrm{hkl})$. Usage of a( $\varphi, \psi, \mathrm{hkl})$ would be necessary when different (hkl)s are used (see e.g. [5,6,7]). If one uses Voigt's or Mandel's notation, we obtain Eq. 3. In the following, we will write all equations in either the one index or the two index notation without further indication.

$$
\sigma_{\mathrm{m}}=\mathrm{b}_{\mathrm{mn}} \mathrm{y}_{\mathrm{n}}
$$

This array of equations is the key for all the error calculations needed since here we can use the law of error propagation.

\section{Error Calculation for $\boldsymbol{\sigma}_{\mathrm{ij}}$}

Using Eq. 3 and the law of error propagation we can write:

$$
\Delta^{2} \sigma_{\mathrm{m}}=\left(\mathrm{b}_{\mathrm{m} 1} \Delta \mathrm{y}_{1}\right)^{2}+\left(\mathrm{b}_{\mathrm{m} 2} \Delta \mathrm{y}_{2}\right)^{2}+\left(\mathrm{b}_{\mathrm{m} 3} \Delta \mathrm{y}_{3}\right)^{2}+\ldots
$$

\section{Error Calculation for the Principal Stresses}

If the principal stress axes are known the calculation of the principal stresses is simply a tensor transformation.

$$
\begin{aligned}
& \sigma_{\mathrm{ij}}^{\mathrm{P}}=\mathrm{t}_{\mathrm{ik}} \mathrm{t}_{\mathrm{j} 1} \sigma_{\mathrm{kl}} \\
& \sigma_{\mathrm{I}}=\sigma_{11}^{\mathrm{P}} ; \sigma_{\mathrm{II}}=\sigma_{22}^{\mathrm{P}} ; \sigma_{\mathrm{III}}=\sigma_{33}^{\mathrm{P}}
\end{aligned}
$$

$\mathrm{t}_{\mathrm{ik}}$ means the transformation matrix, its lines are unit vectors in the directions of the principal axes. Obviously Eq. 5 can also be applied to Eq. 3. This means that $b_{i j n}$ or $b_{m n}$ transform to $b^{\mathrm{P}_{\text {ijn }}}$ or $b^{\mathrm{P}}{ }_{m n}$ in the same way as $\sigma_{\mathrm{ij}}$ or $\sigma_{\mathrm{m}}$ to $\sigma_{\mathrm{ij}}^{\mathrm{P}}$ or $\sigma_{\mathrm{m}}^{\mathrm{P}}$. How this can be done is described below. With $\mathrm{b}_{\mathrm{mn}}^{\mathrm{P}}$ we can write

$$
\Delta^{2} \sigma_{m}^{\mathrm{P}}=\left(\mathrm{b}_{\mathrm{m} 1}^{\mathrm{P}} \Delta \mathrm{y}_{1}\right)^{2}+\left(\mathrm{b}_{\mathrm{m} 2}^{\mathrm{P}} \Delta \mathrm{y}_{2}\right)^{2}+\left(\mathrm{b}_{\mathrm{m} 3}^{\mathrm{P}} \Delta \mathrm{y}_{3}\right)^{2}+\ldots
$$

Here it must be pointed out that although the components $\sigma_{i j}=0$ for $\mathrm{i} \neq \mathrm{j}$ their standard deviations are always larger than zero. Therefore it would be wrong to write: 
$\left(\begin{array}{ccc}\sigma_{I} \pm \Delta \sigma_{I} & 0 \pm 0 & 0 \pm 0 \\ 0 \pm 0 & \sigma_{I I} \pm \Delta \sigma_{I I} & 0 \pm 0 \\ 0 \pm 0 & 0 \pm 0 & \sigma_{I I I} \pm \Delta \sigma_{I I I}\end{array}\right)$

Instead one must write

$$
\left(\begin{array}{ccc}
\sigma_{I} \pm \Delta \sigma_{I} & 0 \pm \Delta \sigma_{12} & 0 \pm \Delta \sigma_{13} \\
0 \pm \Delta \sigma_{12} & \sigma_{I I} \pm \Delta \sigma_{I I} & 0 \pm \Delta \sigma_{23} \\
0 \pm \Delta \sigma_{13} & 0 \pm \Delta \sigma_{23} & \sigma_{I I I} \pm \Delta \sigma_{I I I}
\end{array}\right)
$$

This statement does not apply to the case when one or more than one stress component is set to zero as the consequence of an extra condition, for example a stress free surface or the determining of a principal stress axes due to physical or symmetry reasons. In other words, for a stress component which is not calculated we can also not calculate a standard deviation.

\section{About the Scatter of Principal Stress Axes}

The directions of principal stresses can be very poorly defined or can be completely undefined, even if $\sigma_{\mathrm{ij}}$ are measured with high accuracy. With poorly defined we mean that a small change in one of the $\sigma_{\mathrm{ij}}$ can cause a large change in the directions of the principal axes. This happens in the following cases:

a) $\sigma_{11} \approx \sigma_{22} \approx \sigma_{33} ; \quad \sigma_{12}, \sigma_{13}, \sigma_{23}<<\sigma_{11}$

b) $\sigma_{11} \approx \sigma_{22} ; \quad \sigma_{12}, \sigma_{13}, \sigma_{23}<<\sigma_{11}$

In [1], the authors deal a lot with the question of the scatter of principal axes. In our opinion this is not necessary. Principal axes alone have no physical meaning they describe the stress state only in combination with the principal stresses.

\section{Error Calculation for the Deviatoric Stresses}

The deviatoric stress tensor is defined as

$$
\begin{aligned}
& \sigma_{i j}{ }^{\prime}=\sigma_{i j}-p \\
& p=\operatorname{tr}\left(\sigma_{i j}\right) / 3
\end{aligned}
$$

If we define:

$$
\mathrm{b}_{\mathrm{mn}}^{\mathrm{D}}=\mathrm{b}_{\mathrm{mn}}-\left(\mathrm{b}_{1 \mathrm{n}}+\mathrm{b}_{2 \mathrm{n}}+\mathrm{b}_{3 \mathrm{n}}\right) / 3
$$

we can write for the stress deviator tensor

$$
\sigma_{m}^{\prime}=b_{m n}^{D} y_{n}
$$

Instead of Eq. 4 we then have:

$$
\begin{aligned}
& \left.\Delta^{2} \sigma_{\mathrm{m}}^{\prime}=\left(\mathrm{b}_{\mathrm{m} 1}^{\mathrm{D}} \Delta \mathrm{y}_{1}\right)^{2}+\left(\mathrm{b}_{\mathrm{m} 2}^{\mathrm{D}} \Delta \mathrm{y}_{2}\right)^{2}+\mathrm{b}_{\mathrm{m} 3}^{\mathrm{D}} \Delta \mathrm{y}_{3}\right)^{2}+\ldots \quad \mathrm{m}=1,2,3 \\
& \Delta^{2} \sigma^{\prime}{ }_{m}=\Delta^{2} \sigma_{m} ; \quad m=4,5,6
\end{aligned}
$$

\section{Error Calculation for the Tresca Stress}

For the Tresca yield criterion (maximum shear stress) one needs the largest difference between principal stresses. Since usually principal stresses are ordered according to their magnitude we can write 


$$
\sigma^{\mathrm{T}}=\sigma_{\mathrm{I}}-\sigma_{\mathrm{III}}
$$

and

$$
\Delta^{2} \sigma^{\mathrm{T}}=\left(\left(\mathrm{b}_{11}^{\mathrm{P}}-\mathrm{b}_{31}^{\mathrm{P}}\right) \Delta \mathrm{y}_{1}\right)^{2}+\left(\left(\mathrm{b}_{12}-\mathrm{b}_{32}^{\mathrm{P}}\right) \Delta \mathrm{y}_{2}\right)^{2}+\left(\left(\mathrm{b}_{13}-\mathrm{b}_{33}^{\mathrm{P}}\right) \Delta \mathrm{y}_{3}\right)^{2}+\ldots
$$

\section{Error Calculation for the von Mises Stress}

A frequently used yield criterion was proposed by von Mises. The Mises stress is defined as $\sqrt{ } 3$ times the second invariant of the deviatoric stress tensor.

$$
\sigma^{\mathrm{M}}=\left(3 / 2 \sigma_{\mathrm{ij}}^{\prime} \sigma_{\mathrm{ij}}^{\prime}\right)^{0.5}
$$

We again use the error propagation law. But here it is a little bit more complicated since one must use the derivatives of Mises-stress with respect to the measured values $y_{n}$.

$$
\Delta^{2} \sigma^{\mathrm{M}}=\left(\mathrm{d} \sigma^{\mathrm{M}} / \mathrm{dy}_{1}\right)^{2} \Delta^{2} \mathrm{y}_{1}+\left(\mathrm{d} \sigma^{\mathrm{M}} / \mathrm{dy}_{2}\right)^{2} \Delta^{2} \mathrm{y}_{2}+\left(\mathrm{d} \sigma^{\mathrm{M}} / \mathrm{dy}_{3}\right)^{2} \Delta^{2} \mathrm{y}_{3}+\ldots
$$

The derivative $\mathrm{d} \sigma^{\mathrm{M}} / \mathrm{dy}$ are calculated from Eq. 17 together with Eqs. 9, 10.

$$
\mathrm{d} \sigma^{\mathrm{M}} / \mathrm{dy}_{\mathrm{n}}=0.75 / \sigma^{\mathrm{M}}\left(\sigma^{\prime}{ }_{\mathrm{o}} \mathrm{b}^{\mathrm{D}}{ }_{\text {on }}\right)
$$

\section{Calculation of the Factors $b_{\mathrm{mn}}$}

In the matrix method we have to distinguish between two main cases, and within these there is also some subdivision necessary.

1) The specimen has a stress free surface. $\sigma_{\mathrm{i} 3}=0$

Then, the basis of the whole procedure is Eq.20, 21 [5,6,7]

$$
\mathrm{a}(\varphi, \psi, \text { hkl })=\mathrm{a}_{0}+\mathrm{F}_{11}(\varphi, \psi, \text { hkl }) \mathrm{a}_{0} \sigma_{11}+\mathrm{F}_{22}(\varphi, \psi, \text { hkl }) \mathrm{a}_{0} \sigma_{22}+2 \mathrm{~F}_{12}(\varphi, \psi, \text { hkl }) \mathrm{a}_{0} \sigma_{12}
$$

In the one index notation after Voigt it reads

$$
\mathrm{a}(\varphi, \psi, \mathrm{hkl})=\mathrm{a}_{0}+\mathrm{F}_{1}(\varphi, \psi, \text { hkl }) \mathrm{a}_{0} \sigma_{1}+\mathrm{F}_{2}(\varphi, \psi, \text { hkl }) \mathrm{a}_{0} \sigma_{2}+\mathrm{F}_{6}(\varphi, \psi, \text { hkl }) \mathrm{a}_{0} \sigma_{6}
$$

1.1) $\sigma_{\mathrm{m}}$ are unknown for $m=1,2,6$

With enough measured data, one can create a system of linear equations, the solution of it is:

$$
\begin{aligned}
& \mathrm{a}_{0}=\mathrm{k}^{\dagger}{ }_{\mathrm{nn}} \mathrm{a}_{\mathrm{n}} \\
& \mathrm{a}_{0} \sigma_{1}=\mathrm{k}^{\dagger}{ }_{2 \mathrm{n}} \mathrm{a}_{\mathrm{n}} \\
& \mathrm{a}_{0} \sigma_{2}=\mathrm{k}^{\dagger} \mathrm{an}_{\mathrm{n}} \\
& \mathrm{a}_{0} \sigma_{6}=\mathrm{k}^{\dagger}{ }_{4 \mathrm{n}} \mathrm{a}_{\mathrm{n}}
\end{aligned}
$$

$\mathrm{k}_{\mathrm{mn}}^{\dagger}$ are the entries of the pseudoinverse belonging to that system of linear equations. For further details we refer to $[5,6]$.

By comparing the equations above (Eqs. 22 to 25) with Eq. 3 we see

$$
\mathrm{b}_{1 \mathrm{n}}=\mathrm{k}_{2 \mathrm{n}}^{\dagger} / \mathrm{a}_{0} ; \mathrm{b}_{2 \mathrm{n}}=\mathrm{k}_{3 \mathrm{n}}^{\dagger} / \mathrm{a}_{0} ; \mathrm{b}_{3 \mathrm{n}}=\mathrm{k}_{4 \mathrm{n}}^{\dagger} / \mathrm{a}_{0}
$$

1.2) $\sigma_{6}=0$, which means that the main stress axes are the $x-, y-$, and z-axis.

Then we have only $\sigma_{1}$ and $\sigma_{2}$ as unknowns and only Eq. 22,23,24 are needed, also only $b_{1 n}$ and $\mathrm{b}_{2 \mathrm{n}}$. The error calculations are the same as in the case 1.1, except that principal stresses are already known, therefore also their standard deviations. 
1.3) $\sigma_{1}=\sigma_{2}, \sigma_{6}=0$

Here instead of Eq. 21 one must use Eq. 27

$$
\mathrm{a}(\varphi, \psi, \mathrm{hkl})=\mathrm{a}_{0}+\left[\mathrm{F}_{11}(\varphi, \psi, \mathrm{hkl})+\mathrm{F}_{22}(\varphi, \psi, \mathrm{hkl})\right] \mathrm{a}_{0} \sigma_{11}
$$

For the calculation we again refer to $[5,6]$, the rest is analogous to the considerations above. 1.4) $\sigma_{1} \neq 0$, all other components are zero.

Then $\sigma^{\mathrm{M}}=\sigma^{\mathrm{T}}=\sigma_{\mathrm{I}}=\sigma_{1}$ and all their standard deviations are also known.

For the deviator stress we obtain $\Delta^{2} \sigma_{\mathrm{m}}^{\prime}=\Delta^{2} \sigma_{1}$

2) No stress-free surface. Then Dölle Hauk's equation (Eq. 1) in its original form must be used. The components of the stress tensor can be calculated with Eq. $28 . \mathrm{k}_{\mathrm{mn}}^{\dagger}$ are the entries of the pseudoinverse. For the calculation of them we again refer to $[5,6]$.

$$
\sigma_{\mathrm{m}}=\mathrm{k}_{\mathrm{mn}}^{\dagger} \varepsilon_{\mathrm{n}} \quad \mathrm{m}=1 . .6
$$

We again compare this equation with Eq. 3 to find Eq. 29.

$$
\mathrm{b}_{\mathrm{mn}}=\mathrm{k}_{\mathrm{mn}}^{\dagger}
$$

\section{Transformation of the Matrix $b_{m n}$ to $b^{P}{ }_{m n}$}

The calculation of principal stresses is done with the aid of Eq. 5. If we apply the same procedure to Eq. 3 we immediately obtain the transformation equation for the factors $b^{P}{ }_{m n}$.

$$
b^{P}{ }_{i j n}=t_{i k} t_{j 1} b_{k l n}
$$

Here we have to use the two-indices notation (ij or kl instead of m), this means that at first one must transform $b_{m n}$ to $b_{i j n}$ and after applying Eq. $30 b^{P_{i j n}}$ must be transformed to $b^{P}{ }_{m n}$. Both transformations must follow the schema of Eq. 31. (If Voigt's notation is used the tensors $b_{i j n}$ and $\mathrm{k}_{\mathrm{ijn}}^{\dagger}$ must be dealt with as $\sigma_{\mathrm{ij}}$ whereas $\mathrm{F}_{\mathrm{ij}}$ must be transformed like $\varepsilon_{\mathrm{ij}}$ when changing from one index to two indexes or back.)

$$
\left(\begin{array}{lll}
b_{11 n} & b_{12 n} & b_{13 n} \\
b_{12 n} & b_{22 n} & b_{23 n} \\
b_{13 n} & b_{23 n} & b_{33 n}
\end{array}\right)=\left(\begin{array}{lll}
b_{1 n} & b_{6 n} & b_{5 n} \\
b_{6 n} & b_{2 n} & b_{4 n} \\
b_{5 n} & b_{4 n} & b_{3 n}
\end{array}\right)
$$

\section{Two Special Distributions of Measurement Direction}

For some special configurations of measurement directions, some relationships among different standard deviations are fixed no matter how large standard deviations are.

a) $\varphi_{1}=0^{\circ}, \psi_{1}, \psi_{2}, \ldots \psi_{\mathrm{n}}$

$\varphi_{2}=60^{\circ}, \psi_{1}, \psi_{2}, \ldots \psi_{\mathrm{n}}$

$\varphi_{3}=120^{\circ}, \psi_{1}, \psi_{2}, \ldots \psi_{\mathrm{n}}$

Then we find: $\Delta \sigma_{1}=\Delta \sigma_{2} ; \quad \Delta \sigma_{6}=0.67 \Delta \sigma_{1}$

$\Delta \sigma_{\mathrm{I}}=\Delta \sigma_{\mathrm{II}}=\Delta \sigma_{\mathrm{III}}=\Delta \sigma_{1}$

$\Delta \sigma^{\mathrm{M}} \approx 1.4 \Delta \sigma_{1} ; \quad \Delta \sigma^{\mathrm{T}}=\Delta \sigma_{1}$

b) $\varphi_{1}=0^{\circ}, \psi_{1}, \psi_{2}, \ldots \psi_{\mathrm{n}}$

$\varphi_{2}=45^{\circ}, \psi_{1}, \psi_{2}, \ldots \psi_{\mathrm{n}}$

$\varphi_{3}=90^{\circ}, \psi_{1}, \psi_{2}, \ldots \psi_{\mathrm{n}}$

Then we find: $\Delta \sigma_{1}=\Delta \sigma_{2} ; \quad \Delta \sigma_{6}=\Delta \sigma_{1}$

$0.9 \Delta \sigma_{1} \leq \Delta \sigma_{\mathrm{I}} \leq 1.5 \Delta \sigma_{1}$

$\Delta \sigma^{\mathrm{T}}=1.4 \Delta \sigma_{1} ; \Delta \sigma^{\mathrm{M}} \approx 1.9 \Delta \sigma_{1}$ 
This is another demonstration that the distribution $\varphi=0^{\circ}, 60^{\circ}, 120^{\circ}$ or any other more "symmetric" $\varphi$-distribution is much better than the frequently used distribution $\varphi=0^{\circ}, 45^{\circ}, 90^{\circ}$. See also [2].

\section{Summary}

With the matrix method and its intermediate result, the Moore Penrose pseudoinverse, together with the law of error propagation a tool, not very difficult to handle, has been found to calculate standard deviations of all parameters derived from the stress tensor. In general, all information about the whole measurement procedure must be known. That is: the measurement direction, given by $\varphi / \psi$, the reflection (hkl), the standard deviation $\Delta \mathrm{d}(\varphi, \psi, \mathrm{hkl})$ and the $\mathrm{x}$-ray elastic factor $\mathrm{F}_{\mathrm{ij}}(\varphi, \psi, \mathrm{hkl})$ of each measured lattice plane distance $\mathrm{d}(\varphi, \psi, \mathrm{hkl})$.

\section{References}

[1] D.A. Witte, R.A. Winholtz, S.P. Neal, Counting statistical errors in principal stresses and directions determined by diffraction, Advances in X-Ray Analysis, 37 (1994) 265-267.

[2] B. Ortner, A comparison of different distributions of azimuth angles in thesin ${ }^{2}$ psi-method, Int. J. Mat. Res. (formerly Z.Metallkd.) 98 (2007) 87-90.

[3] H. Dölle, V. Hauk, Einfluß derf mechanischen Anisotropie des Vielkristalls (Textur) auf die röntgenographische Spannungsermittlung, Z. Metallkd. 69 (1979) 410-417.

[4] V. Hauk, Ed. Structural and Residual Stress Analysis by Nondestructive Methods. Amsterdam: Elsevier, 1997

[5] B. Ortner, Properties and general use of the X-ray elastic factors, Advances in X-ray Analysis, Volume 50, (2007) 117-127.

[6] B. Ortner, Why we should give up the $\sin ^{2}$ psi method, Powder Diffaction 24_2-sup (2009) 16_21.

[7] Skrzypek, S.J., et al.,,New approach to stress analysis based on grazing-incidence X-ray diffraction, J. Appl. Cryst. 34 (2001) 427-435. 